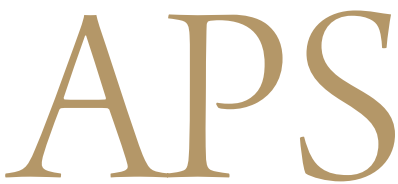

Archives of Plastic Surgery

\title{
The Surgical Release of Dupuytren's Contracture Using Multiple Transverse Incisions
}

\author{
Hyunjic Lee ${ }^{1}$, Surak Eo ${ }^{1}$, Sanghun Cho ${ }^{1}$, Neil F. Jones ${ }^{2}$ \\ ${ }^{1}$ Department of Plastic and Reconstructive Surgery, Dongguk University Ilsan Hospital, Dongguk Graduate School of Medicine, Goyang, \\ Korea; ${ }^{2}$ Hand and Upper Extremity Surgery Center, University of California Irvine, Irvine, CA, USA
}

\begin{abstract}
Dupuytren's contracture is a condition commonly encountered by hand surgeons, although it is rare in the Asian population. Various surgical procedures for Dupuytren's contracture have been reported, and the outcomes vary according to the treatment modalities. We report the treatment results of segmental fasciectomies with multiple transverse incisions for patients with Dupuytren's contracture. The cases of seven patients who underwent multiple segmental fasciectomies with multiple transverse incisions for Dupuytren's contracture from 2006 to 2011 were reviewed retrospectively. Multiple transverse incisions to the severe contracture sites were performed initially, and additional incisions to the metacarpophalangeal (MCP) joints, and the proximal interphalangeal (PIP) joints were performed if necessary. Segmental fasciectomies by removing the fibromatous nodules or cords between the incision lines were performed and the wound margins were approximated. The mean range of motion of the involved MCP joints and PIP joints was fully recovered. During the follow-up periods, there was no evidence of recurrence or progression of disease. Multiple transverse incisions for Dupuytren's contracture are technically challenging, and require a high skill level of hand surgeons. However, we achieved excellent correction of contractures with no associated complications. Therefore, segmental fasciectomies with multiple transverse incisions can be a good treatment option for Dupuytren's contracture.
\end{abstract}

Keywords Dupuytren contracture / Hand / Surgical procedures, operative
Correspondence: Surak Eo Department of Plastic and Reconstructive Surgery, Dongguk University Ilsan Hospital, Dongguk Graduate School of Medicine 27 Dongguk-ro, Ilsandong-gu, Goyang 410-773, Korea

Tel: $+82-31-961-7342$

Fax: +82-31-961-7347

E-mail: surakeo@yahoo.com

Received: 24 Apr 2012 • Revised: 8 Jun 2012 • Accepted: 19 Jun 2012

pISSN: 2234-6163 • elSSN: 2234-6171 • http://dx.doi.org/10.5999/aps.2012.39.4.426 • Arch Plast Surg 2012;39:426-430

\section{INTRODUCTION}

Dupuytren's contracture is characterized by thickening of the palmar fascia and often complicated by cord-like structures that extend from the palm into the affected fingers. Although the cause has not been identified exactly, myofibroblast-mediated nodules and collagen deposits develop the pathogenic cords in the palm. With time, contracture of these cords causes the fingers to flex progressively, resulting in impaired hand function and deformity. Although most commonly seen in older men of northern Eu- ropean descent, Dupuytren's contracture is seen globally across nearly all ethnic groups, with its incidence increasing with advancing age. The highest incidence is in Northern Europe but it is rare in Asia and Africa. Men usually present with an onset 10 years earlier and show higher prevalence of this disease than women [1].

A survey of incidence in Korea has not been performed. However, along with the rise in the geriatric population, the incidence is expected to increase. In the Korean literature, a few surgical approaches for Dupuytren's contracture have been reported.

Copyright $\odot 2012$ The Korean Society of Plastic and Reconstructive Surgeons

This is an Open Access article distributed under the terms of the Creative Commons Attribution Non-Commercial License (http://creativecommons.org/

licenses/by-nc/3.0/) which permits unrestricted non-commercial use, distribution, and reproduction in any medium, provided the original work is properly cited.

www.e-aps.org 
Recently, the surgical treatment with regional fasciectomies using longitudinal incisions and $\mathrm{Z}$-plasties was reported to be popular [2].

Traditionally, contractures were corrected surgically by fasciotomy or aponeurotomy. Recently, however, intralesional injections with a variety of pharmaceutical agents have been attempted in several clinical studies. Even though Xiaflex (Auxilium Pharmaceuticals, Malvern, PA, USA) injection directly into a Dypuytren cord is one method of correcting contracture, clinical studies report that nonsurgical interventions have proved to be largely ineffective and rejected clinically [3].

Surgery continues to be the gold-standard treatment for progressive Dupuytren's contracture. A variety of surgical methods exist and are classified by the amount of diseased tissue removed

\section{Fig. 1. Preoperative design \\ Multiple small transverse incisions drawn on the left hand. If necessary, Bruner zigzag incisions can be added through the dotted line.}

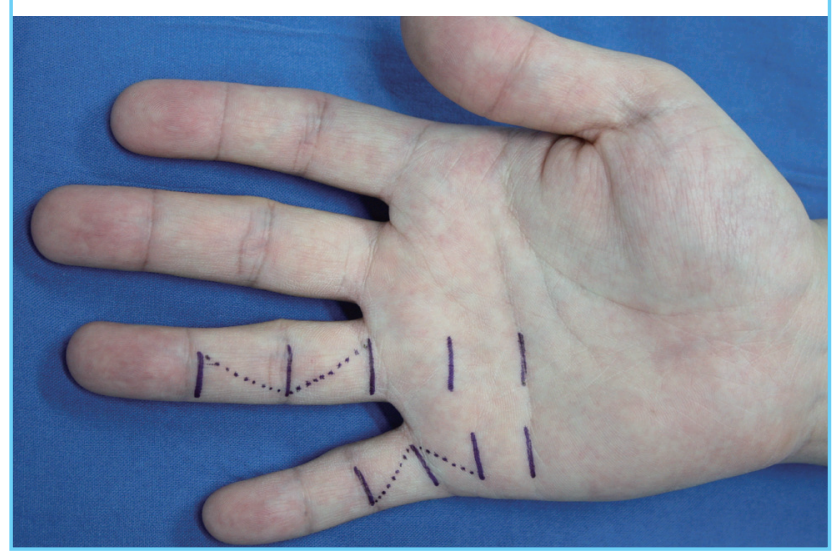

and type of skin incisions. Selective fasciectomy and fasciotomy (excision of all contracted longitudinal fascia in a specific region) is currently the most commonly chosen procedure in preference over total palmar fasciectomy to release of contracture [4]. In choosing a skin incision, longitudinal incisions have the advantage of progressive extensile exposure whereas transverse incisions are less likely to be a pathway for subsequent scar contracture.

Several surgical approaches that have been described in the hand surgery literature require an extensive incision line, and consequently, the incidence of complications is high. Therefore, we introduce a minimally invasive technique with segmental fasciectomies using multiple transverse incisions to reduce the complications from surgical incisions.

\section{IDEA}

Under axillary block anesthesia, a tourniquet was applied to the upper arm at $250 \mathrm{~mm} \mathrm{Hg}$. Transverse incisions (1 to $1.5 \mathrm{~cm}$ ) were typically started over the proximal and distal palmar crease before proceeding distally as necessary to the metacarpophalangeal (MCP) joint crease and the proximal interphalangeal (PIP) joint crease to release the digit into full extension (Fig. 1). After making a space between the skin and diseased cords with a Ragnell retractor, we connected each incision line like tunnels in the subcutaneous layer. To protect the neurovascular bundles, their identification was done initially and special care was taken during dissection from the pathologic cord in the distal palmar incision and in the digital incisions using loupe magnification $(\times 2.5)$. The pretendinous cord was isolated from

\section{Fig. 2. Preoperative and intraoperative findings}

(A) Dupuytren's disease affecting the middle and ring finger with contractures. The metacarpophalangeal (MCP) joint was flexed 120 degrees but the proximal interphalangeal (PIP) joint was not affected in the middle finger. The MCP joint was flexed 90 degrees and the PIP joint was flexed 130 degrees in the ring finger. (B) The fibromatous cords were resected through the transverse incisions. (C) Z-plasties were used to cover the wound of the ring finger. The other incisions were closed directly.
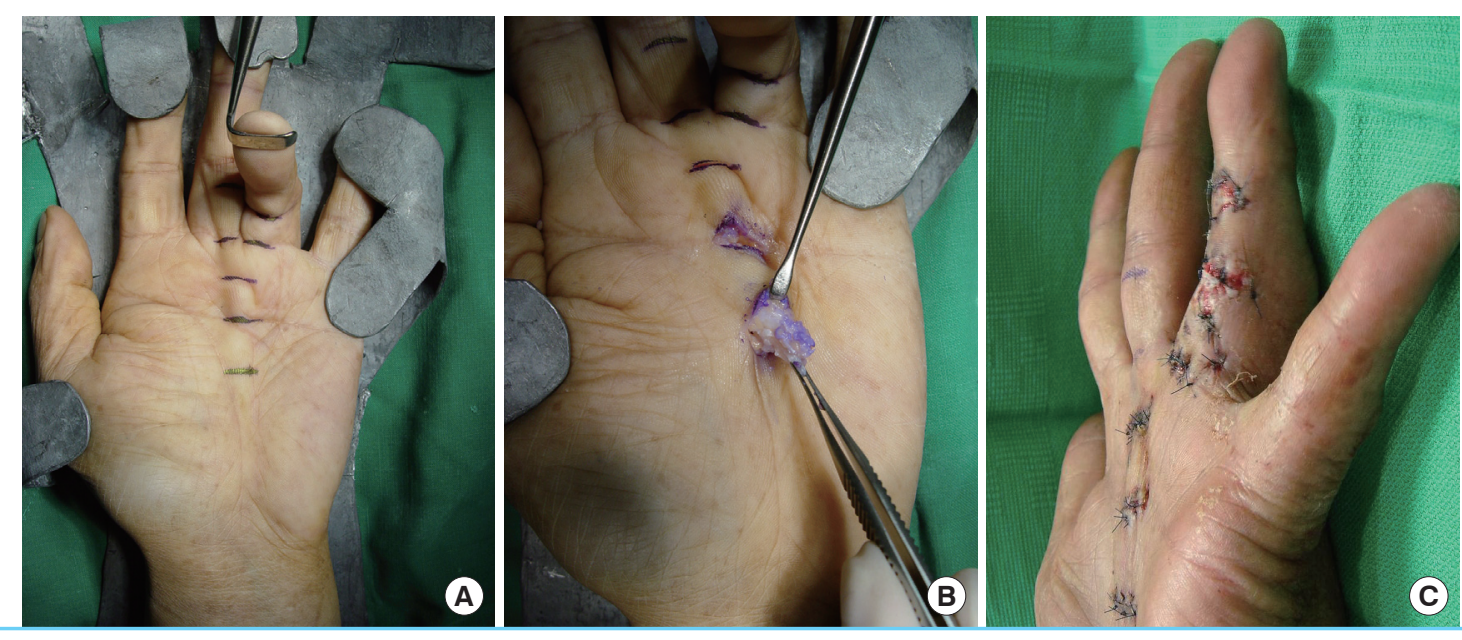
the underlying flexor tendon, and segmental fasciectomies were performed by removing the spiral cords and natatory cords, and mainly the central cords of the affected fingers. We removed the fibromatous cords completely around the Grayson's ligament. By occasionally leaving the intermediate sections of the diseased fascia undisturbed, we could avoid injuring the neurovascular bundles from blind dissection and fasciectomy. By gentle passive manipulation of the joints, the affected digits could be released into full extension. After the tourniquet was deflated, hemostasis was achieved and then the margin of the incisions was loosely approximated with 4-0 nylon. The central portion of the incisions was often left open and was only closed primarily if there was no significant skin tension. One case needed an additional Bruner zigzag incision to cover the skin defect following surgical correction (Fig. 2). The affected digits were splinted in full extension at the MCP joint and PIP joint for 3 weeks postoperatively and night extension splintage for 2 months was followed. A total stitch out was performed at postoperative 3 weeks.

\section{Fig. 3. Preoperative and intraoperative findings}

(A) Dupuytren's disease affecting the little finger with the metacarpophalangeal joint flexed 90 degrees and the proximal interphalangeal joint flexed 110 degrees. (B) The fibromatous cords were resected through the transverse incisions.
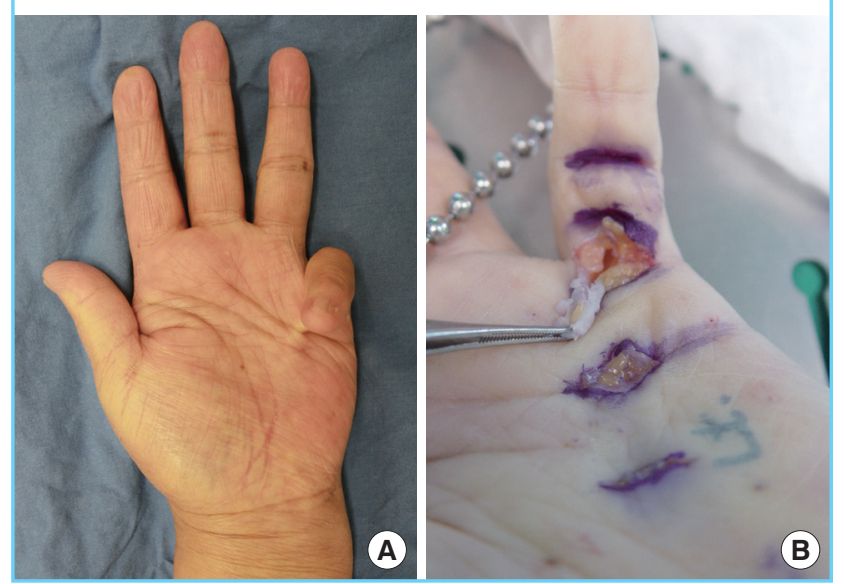

From January 2006 to May 2011, 7 patients were treated with segmental fasciectomy with multiple transverse incisions. Surgical indications were flexion contractures of at least 30 degrees in the MCP or PIP joints and a clearly defined pathologic cord in the palmar fascia (Table 1).

The preoperative mean angle of maximum extension was 100 degrees at the MCP joint and 90 degrees at the PIP joint. All of the 7 patients underwent incisions at the proximal and distal palmar creases and MCP joint creases, and 5 patients at the PIP joint creases. After segmental fasciectomies through multiple transverse incisions, the mean angle of maximum extension improved to 180 degrees at the MCP joint and to 170 degrees at the PIP joint (Figs. 3, 4). All of the patients were followed in outpatient clinics, and the mean follow-up period was 15 months. All patients showed excellent results according to Honner's classification (Table 2) [5].

No postoperative dysaesthesia or sensory deficit was observed and there were no complications such as skin defects or skin necrosis. The patients were prescribed only with nonsteroidal

\section{Fig. 4. Postoperative 18 months}

(A) At postoperative 18 months, there was no flexional contracture and no cords. (B) Full extension was achieved at the metacarpophalangeal and proximal interphalangeal joints.
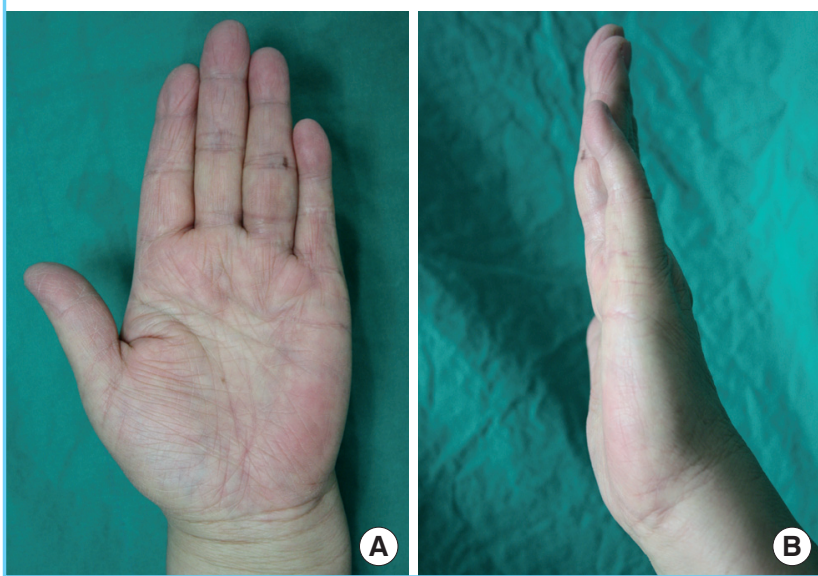

\section{Table 1. Patient information}

\begin{tabular}{|c|c|c|c|c|c|c|}
\hline Case & Age/Sex & Contracture level & $\begin{array}{l}\text { Preoperative maximum } \\
\text { extension angle } \\
\text { (MCP/PIP) }\end{array}$ & $\begin{array}{c}\text { Postoperative } \\
\text { maximum extension } \\
\text { angle (MCP/PIP) }\end{array}$ & $\begin{array}{l}\text { Follow-up } \\
\text { (mo) }\end{array}$ & $\begin{array}{l}\text { Associated } \\
\text { condition }\end{array}$ \\
\hline \multirow[t]{2}{*}{1} & $64 / M$ & Middle finger: MCP & $130 / 180$ & $180 / 180$ & 10 & Ledderhose disease \\
\hline & & Ring finger: MCP \& PIP & $110 / 90$ & $180 / 170$ & 18 & \& Peyronie's disease \\
\hline 2 & $67 / F$ & Little finger: MCP \& PIP & $100 / 80$ & $180 / 180$ & & none \\
\hline 3 & $52 / \mathrm{M}$ & Ring finger: MCP & $90 / 180$ & $180 / 180$ & 16 & none \\
\hline 4 & $65 / M$ & Ring finger: MCP \& PIP & $90 / 100$ & $180 / 160$ & 14 & none \\
\hline 5 & $60 / \mathrm{M}$ & Ring finger: MCP & $90 / 180$ & $180 / 180$ & 24 & none \\
\hline 6 & $71 / \mathrm{M}$ & Ring finger: MCP \& PIP & $100 / 80$ & $180 / 160$ & 12 & none \\
\hline 7 & $62 / F$ & Little finger: MCP \& PIP & $90 / 100$ & $180 / 180$ & 11 & none \\
\hline
\end{tabular}




\begin{tabular}{|l|}
\hline $\begin{array}{l}\text { Table 2. Honner's classification of clinical results of Dupuy- } \\
\text { tren's contracture }\end{array}$ \\
\hline $\begin{array}{ll}\text { Excellent } \\
\text { Good }\end{array}$ \\
$\begin{array}{c}\text { Full flexion and extension of the finger. Full function, no recurrences. } \\
\text { Slight limitation of flexion or extension. Recurrence if present is too } \\
\text { slight to interfere with normal activity. } \\
\text { Limitation of flexion and extension with joint stiffness. Recurrence or } \\
\text { extension limiting function slightly. }\end{array}$ \\
$\begin{array}{l}\text { No improvement on the initial range of movement or function. } \\
\text { Recurrence or extension causing serious loss of function. }\end{array}$ \\
\hline
\end{tabular}

antiinflammatory drugs for 5 days and did not complain of pain. Scar contracture was not observed during the follow-up period.

\section{DISCUSSION}

Felix Plaster first described the flexion contracture of the finger in 1614, and Baron Guillaume Dupuytren reported its suspected pathology, clinical course, and surgical treatment in 1831 [6]. It is a contracture deformity of the hand characterized by the development of new tissue in the form of nodules and cords. This new tissue is of great biologic interest inasmuch as it seems to have some features in common with benign neoplastic fibromatosis and yet undergoes an evolution, through contraction and maturation, similar to wound-healing tissue [7].

The biologic events responsible for Dupuytren's contracture have been studied extensively. New knowledge in this area resulting from the study of the palmar nodules shows the target cells to be the myofibroblasts. These fibroblasts are associated with increased levels of growth factors known to stimulate fibroblasts, such as interleukin-1, basic fibroblast growth factor, transforming growth factor- $\beta 1$ and $-\beta 2$, epidermal growth factor, and platelet-derived growth factor [8].

If functionally significant flexion contractures have not developed, patients may be managed by observation. The presence of a nodule does not always require surgery, and can be softened by injection with triamcinolone. However, a retrospective review showed that $50 \%$ of patients with nodules develop cords [9]. To decide whether patients are good candidates for surgery, placing their hands flat on a table is a good test (Hueston's table top test) [4]. If the MCP joint contractures are more severe than 30 degrees, surgical correction will be of benefit.

The goal of surgery is to restore hand function, not "cure" the disease. To reach this goal, a variety of different surgical options have been described. The least invasive of the surgical interventions, percutaneous fasciotomy [10], is intended to release the tension in the fascia without necessarily removing the diseased fascia. This procedure is associated with recurrent contracture and should be reserved for the few patients who cannot tolerate excision of the diseased fascia. Moermans [11] described a seg- mental fasciectomy in which only short portions of fascia were removed. The advantage of limited fasciectomy is the minimal morbidity associated. However, as the disease progresses, recurrent contracture tends to be a problem. Extensive or radical fasciectomy removes all of the involved fascia with the additional removal of uninvolved fascia to try to prevent disease progression or recurrence [12]. This procedure is good for patients who have extensive disease or increased diathesis. Recurrence is thought to be significantly lowered, but the complication rates are significantly higher.

Choices in skin incision are important for the management of the skin. Longitudinal incisions such as multiple Y-to-V advancement flaps, Bruner [13] zigzag incision, and midline longitudinal incision closed with Z-plasties are more popular and have the advantage of progressive flexible exposure and addressing the skin shortage secondary to the contracture. On the other hand, transverse incisions (long palmar incision and short digital incisions) are advantageous in that they are less likely to be a pathway for subsequent scar contracture. In the open palm technique popularized by McCash [14], transverse skin incisions that had not been amenable to primary closure were managed by full-thickness skin graft closure or by allowing the wound to heal secondarily.

Clibbon and Logan [15] examined 67 patients who underwent segmental fasciectomies through small curved incisions. According to the results of that study, during a follow-up of 2.5 years, there were four patients $(6 \%)$ with recurrence in the MCP joint and seven patients (10\%) with recurrence in the PIP joint. However, in our study, there has been no recurrence in the follow-up period. It is very difficult to compare the recurrence rate between segmental and radical fasciectomies because the range of diseased fascia removed depends on each surgeon. Although it is technically difficult and requires the help of a skilled assistant, complete segmental removal of diseased fascia through the transverse incision could decrease the recurrence rate.

Longitudinal approaches were somewhat invasive techniques used to create a good surgical field and there had been complications like longitudinal scar contracture, postoperative pain, and wound problems. However, our technique of using multiple small transverse incisions is a minimally invasive approach, therefore resulting in only mild postoperative pain and reduction of the healing period. Through tunneling the transverse incisions, we were able to establish a good visual field. After segmental fasciectomy, we could directly approximate the wound with minimal skin tension and infrequently add a Bruner zigzag incision to reduce skin tension, so that no skin defect or skin necrosis was observed. Unlike longitudinal incision, there was no concern about scar contracture affecting finger extension. 
Postoperative complications include loss of flexion, hematoma, skin loss, infection, edema, wound dehiscence, and reflex sympathetic dystrophy. In our cases, there was no hematoma owing to the use of bipolar cautery under loupe magnification and the release of the tourniquet before wound closure.

Splinting is also an important part of postoperative management. Static splints were used after full PIP joint extension had been achieved at the time of operation. Initially, splints should be worn at all times for 3 weeks and removed only for wound care. To prevent wound contracture after stitch out, night time splints should be followed for 8 weeks.

Because only a small number of cases were available and the follow-up periods were not long, our study has limitations. Nevertheless, this study demonstrated that a minimally invasive technique with multiple transverse incisions showed good results regarding the recurrence rate and postoperative complications.

Until recently, longitudinal Bruner zigzag incisions had been more popular than transverse incisions because the latter has a greater chance of complications including skin defects and wound dehiscence. However, we could achieve excellent functional and aesthetic results in treatment of Dupuytren's contracture with multiple small transverse incisions. By using a less invasive technique, all of the patients could return to their daily life earlier and did not complain about pain. Considering our excellent results, we believe segmental fasciectomies with multiple transverse incisions could be a good treatment option in Dupuytren's contracture.

\section{REFERENCES}

1. Sladicka MS, Benfanti P, Raab M, et al. Dupuytren's contracture in the black population: a case report and review of the literature. J Hand Surg Am 1996;21:898-9.

2. Ahn HC, Cheon HJ, Kim KC, et al. Dupuytren's contracture felease with straight line incision and multiple Z-plasty. J
Korean Soc Surg Hand 2011;16:35-41.

3. Hurst LC, Badalamente MA. Nonoperative treatment of Dupuytren's disease. Hand Clin 1999; 15:97-107.

4. Hueston JT. Limited fasciectomy for Dupuytren's contracture. Plast Reconstr Surg Transplant Bull 1961;27:569-85.

5. Honner R, Lamb DW, James JI. Dupuytren's contracture. Long term results after fasciectomy. J Bone Joint Surg Br 1971;53:240-6.

6. Elliot D. The early history of Dupuytren's disease. Hand Clin 1999;15:1-19.

7. TomasekJJ, Vaughan MB, Haaksma CJ. Cellular structure and biology of Dupuytren's disease. Hand Clin 1999;15:21-34.

8. Seyhan H, Kopp J, Schultze-Mosgau S, et al. Increased metabolic activity of fibroblasts derived from cords compared with nodule fibroblasts sampling from patients with Dupuytren's contracture. Plast Reconstr Surg 2006;117:1248-52.

9. Reilly RM, Stern PJ, Goldfarb CA. A retrospective review of the management of Dupuytren's nodules. J Hand Surg Am 2005;30:1014-8.

10. Rowley DI, Couch M, Chesney RB, et al. Assessment of percutaneous fasciotomy in the management of Dupuytren's contracture. J Hand Surg Br 1984;9:163-4.

11. Moermans JP. Segmental aponeurectomy in Dupuytren's disease. J Hand Surg Br 1991;16:243-54.

12. Hurst L. Dupuytren's contracture. In: Green D, Hotchkiss R, Pederson WC, editors. Operative hand surgery. 5th ed, New York: Churchill Livingstone; 2005. p. 159-85.

13. Bruner JM. The zig-zag volar-digital incision for flexor-tendon surgery. Plast Reconstr Surg 1967;40:571-4.

14. McCash CR. The open palm technique in Dupuytren's contracture. Br J Plast Surg 1964;17:271-80.

15. Clibbon JJ, Logan AM. Palmar segmental aponeurectomy for Dupuytren's disease with metacarpophalangeal flexion contracture. J Hand Surg Br 2001;26:360-1. 